\title{
Subject Index Vol. 3, 2006
}

\section{Hour. \\ $=$ \\ Diseases}

Actin 207

ADAM10 255

Adult neurogenesis 12

Alzheimer's disease 134, 197, $227,233,239,247,262,275$, $290,298,313,320,338$

- $\quad$ therapy 305

Amyloid A $\beta 270$

- peptide 275,298

- precursor protein 233, 262, 270, 305

- - protein, knockout 134

- - -, knockdown 134

- precursor-like protein 239

Amyloid- $\beta 305$

$\beta$-Amyloid 334

$\beta$-Amyloid precursor protein 247

- $\quad$, in vivo function 239

Angiogenesis 87

Animal model of Huntington's disease 12

Antisense technology 134

Apoptosis 50

Aspartic protease 290

Astrocyte-like cells 12

Axonal transport 218

BACE inhibitors 290

Basement membrane 56

Brain development 68

Caenorhabditis elegans 227

Cargo receptor 218

$\beta$-Catenin 76

Cell fusion 32

- replacement mechanisms 101

- transplantation 62

CHMP2B 129

Cholesterol 255, 305

Cholinergic interneuron 27

Chromatin remodelling 32

Chromosome 3129

c-Jun N-terminal kinaseinteracting protein 218

2-DE map 112

Dopaminergic cells 45

- neurons 5

Drosophila 338
Ectodomain shedding 262

Embryonic stem cell 32

Endocytosis 262

Endophilin 262

Entactin 56

Epidemiology 320

Epidermal growth factor 38

Epilepsy 56

Epithelial keratin expression 38

Erythropoiesis 68

Erythropoietin $68,87,94$

- receptor 94

Expressed sequence tag scan 106

Fibroblast growth factor 238 - _ - receptors 38

Fragile X-associated tremor/ ataxia syndrome 338

Frontotemporal dementia 129

Functional redundancy 239

Glial fibrillary acidic protein 112

Glycosphingolipids 247

Haplotype H1 327

Hereditary spastic paraplegia 338

Homodimerization 270

Human neurospheres 38

- umbilical cord blood 19

- _ cord blood cells 101

Hyperpolarization-activated cyclic nucleotide-gated current 27

Hypoxia 50, 87

Hypoxic stress 68

Ibuprofen 298

IDE 320

Infectivity 123

Input resistance 27

Insulin 320

Ischemia 87

Ischemic stroke 101

Kinesin 218

Knockout mice 239

Light chain 123

Low-density lipoprotein receptor-related protein 233
Macrophage 334

MAPT 129

- polymorphisms 327

Mass spectrometry 112

Massively parallel signature sequencing 106

Mesenchymal stromal cells 101

Microarray 106

MicroBeads 62

Microglia 334

Microtubule-associated proteins 197

Microtubules 207

Modifier screen 227

Nanog 32

Neural development 5, 76

- progenitor cells 101

- progenitors 76

- stem cell line 19

- $\quad$ cell(s) 38, 106

Neurodegeneration 12, 338

Neurofibrillary tangles, ubiquitinated 313

Neurogenesis 50, 87

Neurogenic niche 12

Neuroinflammation, early stages 313

Neuronal differentiation 5, 19 , 112

- regeneration 87

- stem cells 112

Neuroprotection 87, 338

Nicastrin-like protein 284

Nodal modulator 284

- signaling pathway 284

Non-erythroid cells 94

Non-small cell lung carcinoma cell lines 94

Nonsteroidal antiinflammatory drugs 298

Ntera-2 cells 101

Nuclear reprogramming 32

Oligomerization 270

p21-activated kinase 207

Paired helical filament 197

Parkinson's disease 45, 327, 338

Peroxiredoxin 112

Phosphorylation of tau 207

Photochemical lesion 62
Polyglutamine diseases 338

Polymorphism 334

Presenilin 227, 275, 284, 290

Prion 123

Progenitor cells 106

Proteome profiling 112

Pyramidal neuron 27

Rat hippocampus 50

Reconstructive transplantation strategies 45

Retinoic acid 255

RNA 338

Scrapie 123

Secretase 290, 305

$\alpha$-Secretase(s) 255, 262

$\beta$-Secretase 270

$\gamma$-Secretase 275, 298

Serial analysis of gene expression 106

Signal pathways 76

- peptide peptidase 284

Small interfering RNA technology 134

Sphingomyelin 305

Spinal cord development 38

- _ injury 62

- muscular atrophy 338

Statins 305

Stem cell therapy 87

- - transplantation 101

- cells 5,45

Stroke 62, 87

Subcellular trafficking 247

Subependymal zone 12

Subthreshold membrane resonance 27

Tau protein 197, 327

Transgelin 112

Transgenic mice 56

Urine 123

Urodele amphibian 38

Ventral midbrain 5

Wnt molecules 76

Wnts 5 